\title{
Relation between postoperative blood-aqueous barrier damage and LOCS III cataract gradings following routine phacoemulsification surgery
}

\author{
P G Ursell, D J Spalton, K Tilling
}

\begin{abstract}
Aim-To examine the relation between cataract density, the phacoemulsification energy required for its removal, and blood-aqueous barrier (BAB) damage on the first day after surgery.

Methods-A prospective study recruiting patients with normal eyes apart from senile cataract. Preoperatively, visual acuity, anterior chamber laser flare, and cell values were measured using the Kowa laser flare meter, and the LOCS III grading of the cataract defined. The patients all underwent standardised phacoemulsification surgery through a superior scleral tunnel incision, with confirmed in the bag placement of a one piece PMMA IOL; all surgical complications were excluded. The phaco power delivered was recorded as the cumulative delivered energy, CDE. On day 1 visual acuity and laser flare and cell readings were taken.

Results-101 patients were recruited and all completed the protocol. Older patients had a higher preoperative flare value $(p=0.003)$; preoperative cell values were significantly related to nuclear opacity $(p=0.021)$ and colour $(p=0.011)$. Postoperative flare was related to preoperative flare $(p=0.001)$ and nuclear colour $(p=0.038)$. CDE was related to nuclear colour $(p=0.031)$ and opacity $(p=0.022)$, but not to aqueous flare.

Conclusions-Damage to BAB after routine phacoemulsification surgery is predicted by the preoperative flare values and the density of the cataract nucleus. The amount of phacoemulsification energy required to remove a cataractous lens is related to the density of the cataract but affects postoperative flare to a minimal degree. Other factors such as surgical technique are probably more important. Laser photometry is a useful tool for objectively assessing surgical technique. (Br F Ophthalmol 1997;81:544-547)
\end{abstract}

Excessive disruption of the blood-aqueous barrier (BAB) following cataract surgery delays visual recovery and is associated with the need for prolonged medication. It also increases the risks of postoperative complications such as glaucoma, cystoid macula oedema, pupillary adhesions, and posterior capsular opacification. The laser cell flare meter (Kowa, Japan) provides a rapid, accurate, sensitive, and non-invasive method for assessment of $\mathrm{BAB}$ in the normal and pseudophakic eye by measuring aqueous flare and cells. ${ }^{1-3}$ The LOCS III cataract grading system can be used to classify cataractous changes in the lens, by comparing the patient's lens to standard pictures and grading the cataract for nuclear colour, nuclear opacity, cortical opacity, and posterior opacity. ${ }^{4}$ We have performed a prospective study, using the laser cell flare meter to analyse BAB changes after routine phacoemulsification surgery in normal cataractous eyes which provides an insight into some of the factors contributing to the surgical trauma to the eye, in particular the relation between preoperative flare and cell measurements, the LOCS III classification and the amount of phaco energy required to remove the cataract, and the degree of $\mathrm{BAB}$ damage seen on the first day after surgery.

\section{Methods}

Patients were recruited from those undergoing routine cataract surgery after approval from the hospital ethics committee was obtained. The inclusion criterion for the study was the presence of senile cataract in patients over 60 years of age in an otherwise normal eye. Exclusion criteria were a history of previous intraocular surgery, laser treatment, or ocular disease; or diabetes mellitus requiring medical control, glaucoma, previous uveitis, or any significant posterior segment pathology which would preclude a postoperative vision of $6 / 12$ or better. Patients using topical medications (apart from lubricants) and any patients taking systemic steroids were excluded. Patients who had cataract surgery to the contralateral eye in the previous 4 months were excluded as well as those who were unable to give informed consent.

All patients were seen preoperatively by the same person (PGU). The patient's age and sex were recorded, the iris colour was assessed to be either blue, blue/brown, brown, Hispanic, Asiatic, or Afro-Caribbean which follows the bulk and pigmentation of the iris in increasing order.

LogMAR visual acuity was measured preoperatively and postoperatively using a self illuminated ETDRS chart at 4 metres. The pupil was dilated using phenylephrine 10\% and tropicamide eyedrops $1 \%$ and the cataract was classified into four individual variables by biomicroscopy using the LOCS III grading system, comparing with standard photographs. This system scores the degree of nuclear opac-
Accepted for publication 18 March 1997 
ity, nuclear colour, posterior subcapsular opacity, and cortical opacity in each lens. Nuclear opacity and nuclear colour were graded with a slit beam at $30^{\circ}$ between 0 (no opacity) and 6.9 (maximum opacity) and cortical and posterior subcapsular opacity with retroillumination between 0 (no opacity) and 5.9 (maximum opacity). Anterior chamber (AC) flare and cells were measured using a Kowa FC 1000 laser cell flare meter following pupil dilatation. Seven readings were taken with a variation of $<15 \%$ between background readings, the two extreme values were discarded and the resulting mean and standard deviation calculated.

Patients were operated on within 2 weeks of being assessed by one of three experienced phaco surgeons. All phacoemulsification procedures were performed with the Alcon Master series 10000 through a superior scleral tunnel incision. Sodium hyaluronate was used as the viscoelastic in all cases. After a continuous curvilinear capsulorhexis (CCC), the cataract was removed using either a divide and conquer or a phaco chop technique and a one piece non-surface modified PMMA IOL was placed into the capsular bag. The irrigating solution used was room temperature BSS (Alcon), with $10 \mathrm{mg}$ vancomycin and $0.5 \mathrm{ml} \mathrm{1/1000}$ adrenaline added to $500 \mathrm{ml}$ of solution.

The measurement of 'cumulative delivered energy' (CDE), which is a calculated figure derived from the phaco power administered and the time that the phaco probe is activated, was recorded for each patient. All patients were verified postoperatively to have in the bag placement of the IOL with an intact CCC and no rim tears. Any patient suffering capsular or zonular rupture, or vitreous loss was excluded. No preoperative or perioperative steroids or non-steroidal anti-inflammatory drugs were used and no medication was given before the postoperative assessment visit.

The patients were all re-examined on the first postoperative day between 20 and 28 hours after surgery. LogMAR visual acuity was assessed with a pinhole and the anterior chamber laser flare and cells were measured through a dilated pupil.

\section{STATISTICS}

The relation between preoperative variables (visual acuity, cells, and flare) and the LOCS III cataract grading (nuclear opacity and nuclear colour, posterior subcapsular and cortical opacity) and CDE was examined using linear regression. Each preoperative variable had a model including all LOCS III cataract variables fitted. Backwards stepwise elimination regression was used to obtain a model in which all the variables were significant at the $5 \%$ level. A similar procedure was used to investigate the relations between postoperative variables (visual acuity, flare, and cells) and preoperative and LOCS III variables, and also between patient variables (sex, age, iris colour, and first or second eye operated on) and preoperative and postoperative variables.
Table 1 Preoperative continuous variables

\begin{tabular}{llll}
\hline & Mean & SD & Total \\
\hline Nuclear opacity $^{\star}$ & 3.90 & 1.42 & 96 \\
Nuclear colour $^{\star}$ & 3.92 & 1.49 & 96 \\
Cortical opacity $^{\star}$ & 1.09 & 1.77 & 33 \\
Posterior opacity & 1.05 & 1.62 & 33 \\
Axial length $(\mathrm{mm})$ & 22.89 & 1.11 & 101 \\
Preop vision $\dagger$ & 0.703 & 0.269 & 101 \\
Preop flare (photon count/ms) & 7.72 & 4.14 & 101 \\
Preop cells per $0.075 \mathrm{~mm}^{3}$ & 2.87 & 6.40 & 101 \\
\hline
\end{tabular}

${ }^{\star}$ LOCS III grading.

†LogMAR vision 0.7 is equivalent to $20 / 100$.

Table 2 Preoperative variables

\begin{tabular}{ll}
\hline Sex: & \\
Male & 36 \\
Female & 65 \\
Iris colour: & \\
Blue & 39 \\
Blue/brown & 28 \\
Brown & 15 \\
Race: & \\
Asiatic & 5 \\
Afro-Caribbean & 11 \\
Hispanic & 0 \\
1st/2nd eye: & \\
1st & 58 \\
2nd & 40 \\
\end{tabular}

$\star$ Data unavailable on three cases.

Table 3 Day 1 continuous variables (total 101)

\begin{tabular}{lll}
\hline & Mean & $S D$ \\
\hline Vision $^{\star}$ & 0.315 & 0.159 \\
Flare (photon count $/ \mathrm{ms})$ & 25.58 & 19.79 \\
Cells per $0.075 \mathrm{~mm}^{3}$ & 9.43 & 10.66 \\
\hline
\end{tabular}

${ }^{\star} \operatorname{LogMAR}$ vision 0.3 is equivalent to $20 / 40$.

\section{Results}

In all, 101 patients completed the protocol. There were no cases of corneal oedema on day 1 sufficient to prevent accurate laser cell flare measurements. The preoperative continuous variables are recorded in Table 1 , the discrete preoperative variables are recorded in Table 2, and the results from day 1 are recorded in Table 3.

RELATION BETWEEN LOCS III CATARACT GRADING AND PREOPERATIVE VARIABLES

Nuclear opacity $(p<0.001)$, nuclear colour $(p<0.001)$, and posterior opacity $(p<0.001)$ were significantly related to preoperative visual acuity. Nuclear opacity was significantly related to preoperative $\mathrm{AC}$ cells $(\mathrm{p}=0.021$, $r^{2}=17.4 \%$ ), but not preoperative AC flare; as was nuclear colour $\left(p=0.011, r^{2}=14.8 \%\right)$ when nuclear opacity was excluded from the model. Posterior opacity was significantly related to preoperative AC flare $\left(\mathrm{p}=0.024, r^{2}=3 \%\right)$ but not preoperative AC cells. Cortical opacity was not related to preoperative visual acuity, flare, or cells.

Preoperative AC flare was significantly related to patient age $\left(\mathrm{p}=0.003 r^{2}=8.7 \%\right)$ with older patients having a higher flare value. There was no significant relation between preoperative visual acuity and flare or cells; or between iris colour and flare cells or cataract grading.

RELATION TO DAY 1 LASER FLARE AND CELLS

Day 1 AC flare was highly significantly related to preoperative AC flare $\left(\mathrm{p}=0.001 r^{2}=9.0 \%\right)$. 
Day $1 \mathrm{AC}$ cells were also significantly related to preoperative AC flare $\left(\mathrm{p}=0.021 r^{2}=4 \%\right)$.

Day 1 AC flare was significantly related to nuclear colour $\left(\mathrm{p}=0.038, r^{2}=3.3 \%\right)$ if preoperative flare was not included in the model.

RELATION BETWEEN CDE AND OTHER VARIABLES $\mathrm{CDE}$ was significantly related to nuclear colour $\left(p=0.031, r^{2}=6 \%\right)$ and nuclear opacity $\left(p=0.022, r^{2}=6 \%\right)$, with denser opacities requiring more CDE. There was no significant relation between $\mathrm{CDE}$ and age, postoperative AC flare, cells, or vision.

\section{Discussion}

A reduction in postoperative inflammation is associated with less patient discomfort and reduced requirements for topical medication, faster visual rehabilitation, and potentially a reduction in postoperative complications such as macular oedema and posterior capsule opacification. It is therefore important to minimise surgical trauma to the BAB. Damage to the BAB immediately after cataract surgery can be related to the type of surgery, to incision size, and to the mechanical trauma to the uveal tissues..$^{5-8}$ Within a few days of operation factors such as lens epithelial cell remnant induced $\mathrm{BAB}$ breakdown and inflammation become important. ${ }^{9}{ }^{10}$ Phacoemulsification is less traumatic to the eye than extracapsular surgery. ${ }^{58}$

The laser cell flare meter is currently the most accurate and sensitive method of measuring anterior chamber flare and cells, which directly reflect $\mathrm{BAB}$ damage. Subclinical changes in flare can be followed with speed and accuracy; cell measurements are comparatively less accurate owing to the partial volume effect of measuring a low number of particles in a small volume $\left(0.75 \mathrm{~mm}^{3}\right)$. Measurements are made by measuring the scattering of a $\mathrm{HeNe}$ laser beam by particles in the AC and are easier to make following pupillary dilatation. However, this effectively lowers flare values as a result of a mixing of aqueous from the posterior chamber which has lower protein concentrations compared with that of the anterior chamber; it is therefore important to be consistent with mydriasis.

Preoperative flare measurements in this study were consistent with our previous findings, ${ }^{1}$ and confirm that iris colour does not affect flare values and that AC flare increases with age. This is probably due to an increased concentration of macromolecules in the aqueous rather than an increase in aqueous protein concentration. ${ }^{11}$ Although the laser flare cell meter is optimised to count particles of $10 \mu \mathrm{m}$ diameter other particles such as pigment granules can be counted. Cells and pigment can be liberated from the iris by pupillary dilatation, cataractous lenses are larger than normal lenses, ${ }^{12}$ and the rubbing of the cataractous lens against the iris pigment epithelium may well explain why we found eyes with a greater degree of nuclear opacity had higher levels of cells (or pigment) preoperatively. It is interesting to note that flare was higher in eyes with posterior subcapsular opacity, which is also found in association with conditions, such as uveitis or diabetes, where the BAB is damaged, and it is conceivable that these subclinical $\mathrm{BAB}$ changes might have some aetiological significance in the pathogenesis of posterior subcapsular cataract in otherwise clinically normal eyes.

On the first postoperative day the mean flare value of our patients was 25.6 (SD 19.8) photons/ms (Table 3). Similar flare values following phacoemulsification of 37.8 (2.6) and of approximately 28 have been reported on the first postoperative day. ${ }^{8}{ }^{13}$ Oshika et al reported values of 20.1 (17.3) on day 1 after phaco surgery and found that smaller incision sizes were related to lower flare values. ${ }^{7}$ In our study it is of interest that there was a correlation between pre- and postoperative flare on day 1 following the exclusion of confounding variables (Table 1) although the correlation coefficient was low. This suggests that the uveal vessels of each individual eye have an innate blood-ocular barrier function, so that apparently clinically identical eyes could react differently to the same surgical stimulus. In a normal eye this variation is small but could be more important in eyes where the $\mathrm{BAB}$ has already compromised, as in diabetic or uveitic eyes.

Increasing nuclear colour is widely recognised by cataract surgeons to be an indicator of 'hardness' of the nucleus and a useful observation to make when planning surgical technique, ${ }^{14}$ but we are not aware of any publications where this has been accurately correlated with the amount of phacoemulsification energy required to remove the nucleus. In an in vitro study, lens hardness correlated independently with age and nuclear colour and opalescence when measured by a guillotine cutting technique. ${ }^{15}$ In our study the total energy required to emulsify the cataract, $\mathrm{CDE}$, correlated as expected with nuclear colour and opacity but aqueous flare on the first postoperative day did not. Regression analysis showed that nuclear colour and opacity account for only $12 \%$ of delivered phaco energy, CDE. This implies that nearly $90 \%$ of the CDE used cannot be accounted for by the clinical evaluation of lens hardness, suggesting that a substantial amount of phaco energy is dissipated and not used effectively during surgery with present surgical techniques. Examples of this might include situations such as sculpting, where the phaco needle tip is only partially in contact with the nucleus; or during fragment removal when the fragment does not fully cover the needle tip. Our results suggest that with better surgical techniques or instrumentation design the phaco energy delivered to the eye could be considerably reduced.

Regression analysis shows that preoperative flare and nuclear colour contribute $12.3 \%$ to postoperative flare. This implies that BAB damage is caused by other surgical factors which include incision size, the duration of surgery, the volume of irrigating fluid used, intraocular medication and, probably most importantly, surgical manipulation and iris touch. ${ }^{5-8}$ The fact that harder lenses are associ- 
ated with increased AC flare postoperatively is probably due to the increased manipulation required and the longer operating time. The surgery in our study was carried out by three experienced surgeons and assessment of the relative importance of these factors would require additional work in which these factors are quantified for individual surgeons.

The laser cell fare meter is an effective tool for measuring $\mathrm{BAB}$ damage after phaco surgery and can be used to monitor outcomes. There are applications for this in training and assessing junior surgeons, quality control, and evaluating new surgical techniques.

PGU is sponsored by the Iris Fund for the Prevention of Blindness and a grant from Kowa Ltd.

1 Shah SM, Spalton DJ, Smith SE. Measurement of aqueous cells and flare in normal eyes. $\mathrm{Br} \mathcal{F}$ Ophthalmol 1991;75:348-52.

2 El-Maghraby A, Marzouki A, Matheen TM, Souchek J, van der Karr M. Reproducibility and validity of laser flare/cell meter measurements as an objective method of assessing intraocular inflammation. Arch Ophthalmol 1992;110:960-

2 .

Shah SM, Spalton DJ. Changes in anterior chamber flare and cells following cataract surgery. $\mathrm{Br} \mathcal{F}$ Ophthalmol 1994 78:91-4

4 Chylack LT Jr, Wolfe JK, Singer DM, Leske MC, Bullomore MA, Bailey IL, et al. The lens opacities classification system III. Arch Ophthalmolol 1993;111:831-6.
5 Pande M, Spalton DJ, Kerr-Muir M, Marshall J. Postoperative inflammatory response to phacoemulsificaton and extracapsular cataract surgery: aqueous flare and cells. $\mathcal{F}$ Cataract Refract Surg 1997 (in press).

6 Ferguson VMG, Spalton DJ. Continued breakdown of the blood aqueous barrier following cataract surgery. $\mathrm{Br} \mathcal{F} \mathrm{Oph}$ thalmol 1992;76:453-6.

7 Oshika T, Tsuboi S, Yaguchi S, Yoshitomi F, Nagamoto T, Nagahara K, et al. Comparative study of intraocular lens implantation through 3.2 and $5.5 \mathrm{~mm}$ incisions. Ophthalmology 1994;101:1183-90.

8 Oshika T, Yoshimura K, Miyata N. Post surgical inflammation after phacoemulsificaton and extracapsular extraction with soft or conventional intraocular lens implantation. $\mathcal{F}$ Cataract Refract Surg 1992;18:356-61.

9 Nishi O, Nishi K. Disruption of the blood-aqueous barrier by residual lens epithelial cells after intraocular lens implantation. Ophthalmic Surg 1992;23:325-9.

10 Pande M, Shah SM, Spalton DJ. Correlations between aqueous flare and cells and lens surface cytology in eyes with PMMA and heparin surface modified intraocular lenses. F Cataract Refract Surg 1995;21:326-30.

11 Shah SM, Spalton DJ, Taylor JC. Correlations between laser flare measurements and anterior chamber protein concentrations. Invest Ophthalmol Vis Sci 1992;33:2878-904.

12 Phelps Brown NA, Bron AJ. Lens disorders. A clinical manual of cataract diagnosis. Butterworth-Heinemann, Oxford, 1996.

13 Martin RG, Sanders DR, Van der Karr MA, DeLuca M. Effect of small incision intraocular lens surgery on post operative inflammation and astigmatism - a study of the AMO SI $18 \mathrm{NB}$ small incision lens. F Cataract Refract Surg 1992;18:51-7.

14 Gimbel H. Divide and conquer nucleofractis phacoemulsificaton. Development and variations. F Cataract Refract Surg 1991;17:281-91.

15 Heyworth P, Thompson GM, Tabandeh H, McGuigan S. The relationship between clinical classification of cataract and lens hardness. Eye 1993;7:726-30. 